\title{
La "shadokologie » dans la première et la deuxième série des Shadoks
}

Jessica Kohn

\section{(2) OpenEdition \\ 1 Journals}

Édition électronique

URL : http://journals.openedition.org/aes/332

DOI : 10.4000/aes.332

ISSN : 2258-093X

Éditeur

Laboratoire LISAA

Référence électronique

Jessica Kohn, « La « shadokologie » dans la première et la deuxième série des Shadoks », Arts et Savoirs [En ligne], 5 | 2015, mis en ligne le 29 octobre 2015, consulté le 19 avril 2019. URL : http:// journals.openedition.org/aes/332; DOI : 10.4000/aes.332

Ce document a été généré automatiquement le 19 avril 2019

Centre de recherche LISAA (Littératures SAvoirs et Arts) 


\title{
La « shadokologie » dans la première et la deuxième série des Shadoks
}

\author{
Jessica Kohn
}

1 Créé par Jacques Rouxel, le dessin-animé des Shadoks inscrit l'imaginaire français de la fin des années 1960 dans un contexte technologique et artistique résolument moderne. Cette histoire contée à l'écran par Claude Piéplu prend en effet pour support la télévision, un objet encore relativement neuf et qui commence tout juste à s'imposer comme un élément indispensable des foyers français : en 1968, 63,5 \% des Français en possèdent une. La série est, en outre, produite par le Service de la Recherche, organisme créé en 1960 par Pierre Schaeffer ayant pour but de mener une recherche fondamentale sur l'image et le rapport à la communication de masse, et qui s'est notamment spécialisé dans le cinéma d'animation. Enfin, elle a d'abord été conçue comme un exercice d'animation au service d'une machine-prototype, "l'animographe », permettant de simplifier la création des dessins-animés. Les Shadoks, depuis leur conception théorique et pratique jusqu'à leur diffusion dans les foyers français, s'appuient donc sur les progrès scientifiques et techniques de la fin des années 1960. De février à avril 1969, la première série est d'ailleurs rediffusée sur la deuxième chaîne, dont la particularité est de proposer, depuis 1967, une image en couleurs. J'étudierai ici les deux premières séries qui, outre leur rediffusion mentionnée ci-dessus, sont présentées en avril, mai, septembre et octobre 1968 et de juin à juillet 1970, sur la première chaîne et en noir et blanc, à l'heure d'écoute maximale, c'est-à-dire pendant la minute qui suit les informations télévisées de 19h30. En raison de leur horaire et de leur nouveauté, les deux premières séries ont souvent été vécues par les téléspectateurs français comme une sorte d'attentat culturel.

2 La forme de la série, comme les sujets que celle-ci aborde, font écho à cet arrière-plan moderne et technologique. L'effet visuel, tant du point de vue des formes que des couleurs, est très clairement hérité de l'avant-garde représentée par l'école de l'abstraction viennoise et parisienne, et plus spécifiquement par Klee, Kandinsky et Miro. Si cet héritage n'est pas revendiqué nommément par Jacques Rouxel, c'est parce qu'il s'inscrit dans la lignée d'un cinéma d'animation qui s'oppose après-guerre au style 
anthropomorphique et sphérique de Walt Disney ${ }^{1}$. À ce propos, Rouxel, à la suite de pionniers comme Stephen Bosustow et Piotr Kamler, a également privilégié pour son dessin un style réaliste, aux traits anguleux et aux surfaces plates, directement inspiré par la ligne du dessinateur du New Yorker, Saul Steinberg, un artiste roumaino-américain pionnier en son genre ${ }^{2}$. L'ambiance musicale, quant à elle, est une expérience totalement inédite menée par Pierre Schaeffer autour de la musique concrète, musique qui se sert, ou s'inspire, des sons produits par des objets de la vie quotidienne. Ces éléments de la modernité, issus tant des artistes de l'avant-garde que des pionniers du dessin animé, servent de toile de fond à un feuilleton dont les sujets sont ancrés dans la société contemporaine et ses avancées scientifiques, c'est-à-dire, encore plus que dans la culture de masse, dans la culture médiatique. En utilisant cette dernière expression, nous pouvons mettre l'accent sur ce qui, dans le récit, relève tout autant de la circulation des discours que de la démultiplication des supports destinés à un large public, et qui justifie donc que la narration soit aussi interprétée comme une source historique. ${ }^{3}$ Dans la série, il est question de navigation marine, de progrès de la médecine et des sciences, mais, surtout, de conquête spatiale: c'est un véritable feuilleton cosmique qui est offert au téléspectateur, fait de fusées, de radars, de satellites, d'espionnage industriel, de course à la Terre, et même de course à la Lune.

3 Les épisodes dits de la " course à la Lune » sont écrits en 1969, en pleine guerre froide, et quelques mois à peine avant les premiers pas de l'homme sur la Lune, le 20 juillet. Cependant, malgré ces ressemblances avec les actualités, la plupart des épisodes mettent en scène une cosmologie, une biologie et des technologies abracadabrantes, qui parodient leurs équivalents humains. À ce titre, la question du genre de la série fait très vite surface, et il est tentant de parler soit de science-fiction, soit de futurisme, quoique toujours sur le mode parodique. La référence au futurisme est problématique, tant il est exagéré de relier la création de Rouxel à l'école italienne du début $\mathrm{du} \mathrm{XX}^{\mathrm{e}}$ siècle. Si nous nous donnons malgré tout la liberté de parler à certains moments de conte "futuriste", c'est en reprenant le vocabulaire des téléspectateurs et des commentateurs de l'époque, qui semblent s'attacher à définir ainsi « l'attitude de celui qui se tourne vers l'avenir $»^{4}:$ pour les acteurs d'alors, le feuilleton est perçu comme s'inscrivant résolument dans une sorte de futur qui, à défaut d'être crédible, est du moins potentiel. C'est alors que le terme de science-fiction se révèle indispensable, sinon comme outil de définition exacte du genre de la série, du moins comme adjuvant dans cette entreprise. De fait, l'univers technologique développé à partir d'un référentiel ordinaire confronte le spectateur à des «situations et des événements appartenant à un avenir plus ou moins proche et à un univers imaginé en exploitant ou en extrapolant les données contemporaines et les développements envisageables des sciences et des techniques $»^{5}$. Si la science-fiction n'a pas de définition véritablement fixe ${ }^{6}$, l'attribution générique est, ici, primordiale ${ }^{7}$, à une époque où elle commence à devenir populaire en tant qu'objet culturel.

Outre cette parodie des savoirs scientifiques pratiques, la série s'attaque aux savoirs théoriques et mathématiques, en s'inspirant de la 'Pataphysique, la «science des solutions imaginaires » créée par Alfred Jarry à la fin du XIX siècle. L'ensemble ainsi formé, à la fois pratique, technologique et théorique, pourrait s'appeler "shadokologie ", terme par lequel on désignerait la science qui balaye tous les aspects de la vie des Shadoks, depuis l'univers dans lequel les personnages évoluent jusqu'à la logique qui guide leurs actions, en incluant, pour finir, la science développée sur les Shadoks euxmêmes. Jacques Rouxel cherche en fait moins à produire des allégories de l'actualité 
contemporaine qu'à utiliser un système référentiel commun qui permet de porter le récit auprès des différents publics. Parce qu'il s'appuie de manière presque permanente sur un substrat scientifique, il nous renseigne sur une des obsessions de la fin des années 1960, à une époque où la réalité semble parfois rejoindre la fiction. Cependant, en utilisant un ressort comique qui s'appuie sur le détournement, et qui traite ainsi chaque science imaginaire sous le spectre de l'absurde, il pose aussi la question de l'aliénation créée par la croyance aveugle dans les sciences. C'est peut-être en ce sens que l'on peut comprendre l'abandon de la transmission du savoir à deux figures tutélaires, le professeur Shadoko et le Devin Plombier, dont l'autorité ne paraît guère fondée rationnellement. Les sciences imaginaires sont ici tout autant au service du jeu et de l'imagination que d'un questionnement sur le bien-fondé de l'autorité scientifique, toujours valables une fois l'écran éteint.

\section{Une genèse « futuriste ": les lois scientifiques d'un monde imaginaire}

5 Les Shadoks sont avant tout une œuvre d'imagination farfelue, œuvre que l'on pourrait qualifier de genèse "futuriste", au sens où nous l'entendions en introduction. Chaque invention, aussi loufoque soit-elle, obéit cependant à des lois scientifiques, qui donnent lieu à des exposés en voix-off de la part du narrateur, dont l'érudition est contrebalancée par un vocabulaire simple imitant le niveau de langage enfantin, comme on le constate dès le premier épisode :

C'était il y a très, très, très longtemps. En ce temps-là, il y avait le ciel. À droite du ciel, il y avait la planète Gibi. Elle était complètement plate, et elle penchait, soit d'un côté, soit de l'autre. À gauche du ciel, il y avait la planète Shadok. Elle n'avait pas de forme spéciale, ou plutôt elle changeait de forme. Au milieu du ciel, il y avait la Terre, qui était ronde et qui bougeait. La Terre, où il n'y avait apparemment rien. Sur la planète Gibi il y avait des animaux qui s'appelaient des Gibis. Voici un Gibi vu de près. En voici un autre. Quand il y avait trop de Gibi d'un côté, la planète penchait, les Gibis glissaient, il y en avait qui tombaient. C'était très gênant, surtout pour les Gibis. Sur la planète Shadok, il y avait des Shadoks de deux sortes. Des Shadoks avec des pieds en bas qui vivaient au-dessus de la planète, et des Shadoks, avec des pieds en haut, qui vivaient de l'autre côté, et qui servaient à soutenir la planète par en dessous. Comme la planète changeait de forme il y avait des Shadoks qui tombaient, c'était très gênant, surtout pour les Shadoks. Les Shadoks et les Gibus en eurent donc assez au bout d'un certain temps de vivre sur des planètes qui ne marchaient pas bien, alors ils décidèrent les uns et les autres d'aller sur la Terre, qui avait l'air de mieux marcher. ${ }^{8}$

6 L'histoire des Shadoks a pour toile de fond permanente le cosmos, à la fois objet central des œuvres de science-fiction et au cœur des préoccupations des années 1960. Si on y retrouve certains éléments connus - la Terre, la Lune et les fusées qui permettent de s'y rendre - la plupart sont inventés de toutes pièces. Le voyage jusqu'à la Terre est l'occasion, dans la première série, d'une découverte des autres planètes du cosmos, toutes aussi improbables que celles des Gibis et des Shadoks : la planète à eau, par exemple, est composée uniquement d'eau'. La Terre, rejointe à la fin de la première série, offre elle aussi de pittoresques détails, dont la Lune est un parfait exemple :

En ce temps-là, en effet, sur la Terre, il y avait la Lune. Mais elle était de bien moindre qualité que celle de maintenant, ce n'était pas encore un astre, ni même une planète, ce n'était même pas un satellite de planète. C'était un "machin ", un 
machin qui se promenait à deux ou trois coudées au-dessus des arbres et des forêts, comme un vulgaire ballon de foire. ${ }^{10}$

7 Les particularités biologiques des personnages, Shadoks, Gibis, et Gégène (un insecte petit mais malfaisant, " maître de la terre et de tous ses éléments ${ }^{11}$ ), obéissent aux mêmes lois scientifiques improbables : les Gibis tirent leur intelligence de leur chapeau; les Shadoks, pour se reproduire, doivent compter jusqu'à quatre. Les objets de la vie quotidienne ne sont pas en reste : les passoires et les pompes sont constamment détournées tout au long des deux séries, quitte à devenir un motif graphique de répétition. Parapluies, échelles ou bouteilles subissent le même traitement, qui détourne l'ordinaire : un épisode s'emploie ainsi à distinguer les échelles qui montent des échelles qui descendent. Mais c'est peutêtre la technologie qui tient la plus grande place dans ce feuilleton cosmique. Des fusées jusqu'au « cosmotobus $»^{12}$ en passant par les " graines de radar ${ }^{13}$, toutes les références aux progrès scientifiques modernes sont permises. Du point de vue technologique, ce récit se situe donc en partie dans le futur, parce qu'il extrapole les progrès en cours, dont sont témoins les téléspectateurs français. Après tout, les Shadoks marchèrent sur la Lune avant Armstrong et ses coéquipiers - même s'ils le font sans fusée, et grâce à une échelle. Les commentateurs français ne s'y trompèrent pas, comme le montre le vocabulaire utilisé par la pré-enquête de l'O.R.T.F. en mai 1968 : le feuilleton, selon celle-ci, plaît avant tout aux enfants et aux jeunes qui montrent « un engouement pour ce qui est moderne et nouveau ", pour le "côté futuriste de l'histoire et les références à un monde interplanétaire d'actualité qui les passionne ${ }^{14}$. Les adjectifs utilisés ici sont particulièrement éclairants quant à l'état d'esprit de l'époque en matière de modernité : celle-ci touche au futur, mais est d'ores et déjà incluse dans une actualité qui fait la part belle aux découvertes de l'astronomie, dont les bonds en avant sont alors presque perçus comme une échappée dans l'impossible.

Figure 1. La fusée des Gibis, un exemple de technologie moderne

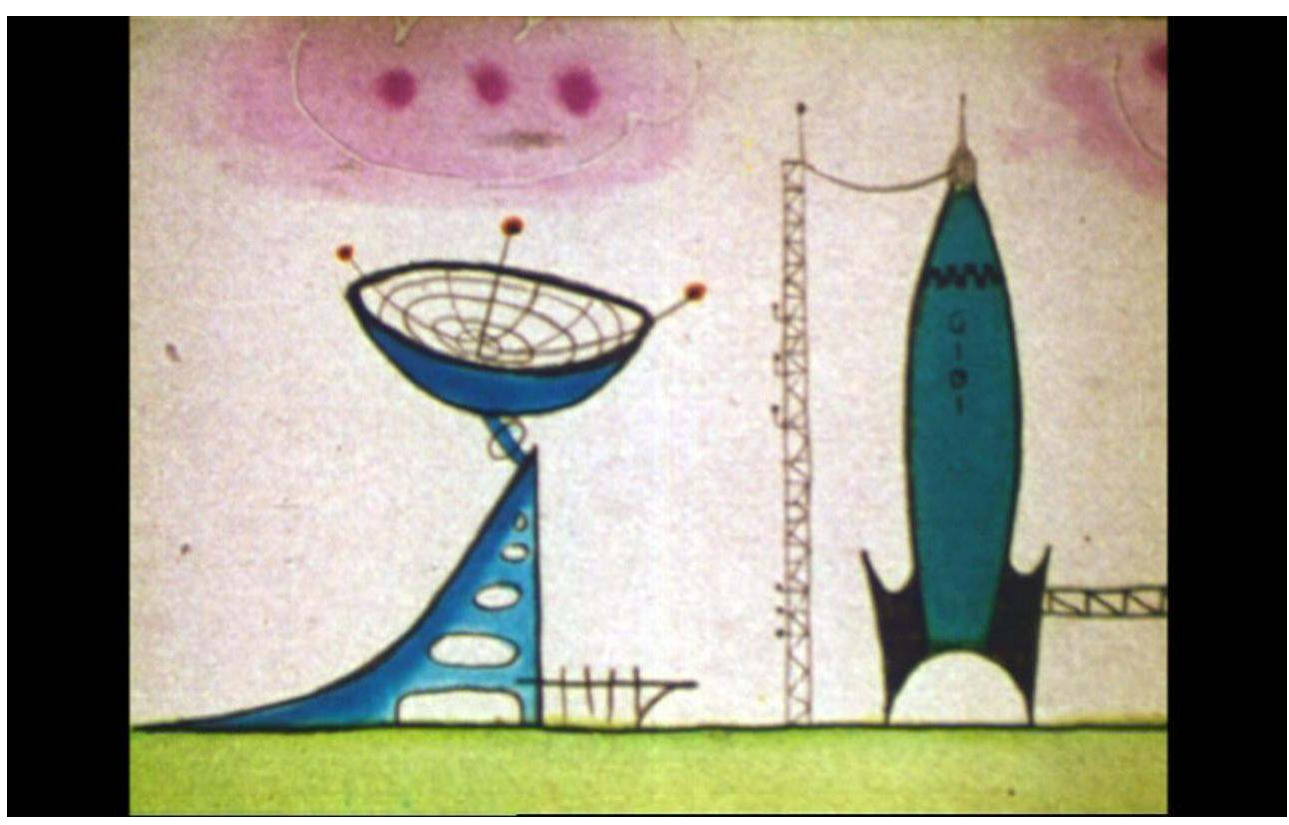

Mais ce récit du futur nous vient également du passé puisque il est très clairement situé avant notre ère, « il y a très, très, très longtemps ». L'ancrage dans le temps, formulé avec le moins d'exactitude possible, à la manière d'un conte, permet justement d'en dérouler 
la temporalité floue, les péripéties et les personnages-types. Les Gibis, tous identiques, ne sont que l'éternelle répétition du type de héros parfait, à la fois bon et intelligent. Les Shadoks, puisqu'imparfaits, présentent au contraire plus de personnalités, mais toujours sous forme de types : le chef Shadok, cruel et autoritaire, le professeur Shadoko, fou et génial, et le Devin Plombier, sorte de sorcier du village. Pourtant, l'atemporalité du conte est loin d'être respectée: le passé du récit précède explicitement le temps des téléspectateurs, (à l'instar de la Lune qui, «en ce temps-là était de bien moindre qualité que celle de maintenant») et permet parfois d'expliciter le monde actuel, comme les lampadaires, les feux rouges, et tous ces objets qui « tombent en panne ou font du mal par vengeance ", qui ne sont que des Shadoks prisonniers des Gibis, transformés en objets ménagers et ensuite oubliés sur la Terre ${ }^{15}$.

Pour permettre d'appréhender ce monde qui ne se situe explicitement dans aucune temporalité et dans aucun univers précis, le récit est entrecoupé d'épisodes "pauses ", faits d'exposés scientifiques censés en justifier les bizarreries, expliquant par exemple comment les Shadoks respirent, ou de quelle manière les Gibis se font des costumes ${ }^{16}$. On peut voir certains de ces procédés explicatifs à l'œuvre dans le premier épisode de la première saison, cité ci-dessus : le narrateur nous montre deux Gibis « vus de près ", et le dessin privilégie le gros plan. Puis, quand il s'agit de présenter les deux sortes de Shadoks qui cohabitent, les images se superposent à la façon de diapositives. Grâce à ces techniques filmiques et discursives, le téléspectateur est désormais à même de comprendre comment fonctionne le nouveau monde auquel il est confronté, composé notamment de deux planètes habitées, mais bancales. Le niveau de vocabulaire explicatif, cependant, à l'instar du dessin, est simplifié et enfantin : les planètes ne «marchent pas bien ", par exemple : la parodie de la scientificité du récit se retrouve à tous les niveaux, du détournement des objets connus des téléspectateurs à l'utilisation d'un langage et d'un dessin qui invoquent les codes de l'exposé scientifique sans les respecter jusqu'au bout. De manière plus générale, Jacques Rouxel aime jouer avec la langue, surtout la langue scientifique qui lui laisse un large éventail de possibilités, notamment à travers l'invention de nombreux néologismes. Ainsi, le carburant qu'utilisent les Gibis pour leur fusée s'appelle-t-il le «Cosmogol $999 \aleph^{17}$. Ce nom est riche de significations : son suffixe en [ol], tout comme l'ajout d'un nombre élevé, sont des procédés courants de désignation du gaz. Tout en étant inventée, cette appellation s'inscrit donc sans mal dans l'univers technologique de la fin des années soixante. À la longue, le feuilleton finit par développer son propre univers scientifico-linguistique, de sorte que certains ressorts métadiégétiques se mettent en place, créant du comique de répétition verbale : après la « shadokaravelle » dans la première série, ce sont les « shadoknautes » qui sont introduits à l'écran dans la série suivante ${ }^{18}$. 
Figure 2. La pratique parodiée de l'exposé scientifique : une fusée enfantine

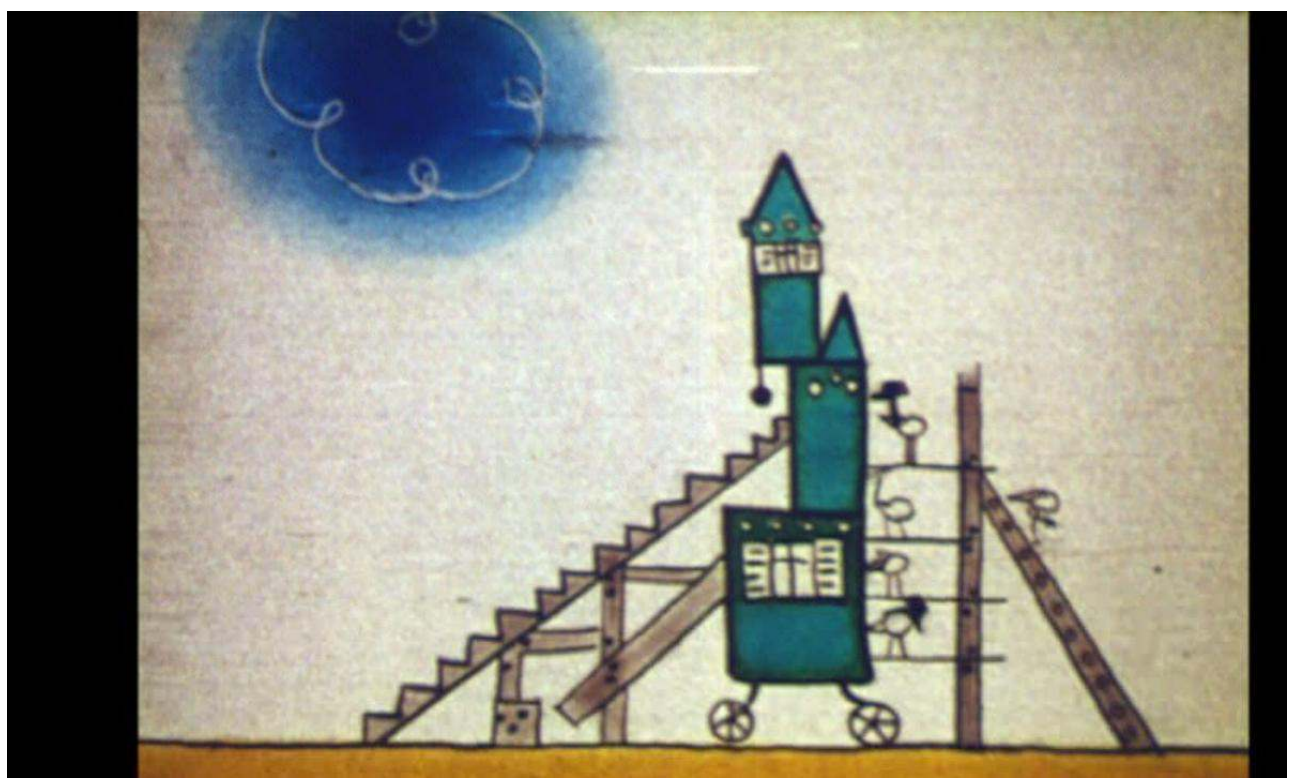

Ainsi se construit un monde improbable, pour trois raisons : d'abord parce qu'on y trouve des planètes, personnages et objets, non seulement inventés, mais également invraisemblables; de plus, les lois de la physique sont sans cesse déjouées, à commencer par la loi de la gravitation ${ }^{19}$; enfin, le dessin lui-même déforme les référents connus du téléspectateur, proposant par exemple une Lune rouge et non jaune. À travers le langage même utilisé pour décrire ce monde incongru, le récit déstabilise sans cesse les connaissances du téléspectateur, tout en se présentant comme en lien avec le monde actuel. Improbable, loufoque, et se référant pourtant à un monde existant : c'est peut-être de cette proximité entre deux forces contraires que naît la logique de l'absurde. L'absurdité du monde induit la narration et ses péripéties: si les Gibis et les Shadoks doivent aller sur la Terre, c'est parce que leurs planètes «ne marchent pas bien $»^{20}$. Inversement, il existe une montagne où l'atmosphère descend assez bas pour permettre aux Shadoks de partir dans l'espace ${ }^{21}$. Nous avons donc affaire à un monde malléable, maléfique ou bénéfique selon les exigences des péripéties. En d'autres termes, le monde et ses lois sont créés en fonction des besoins de l'histoire, et au fur et à mesure de la narration. Mais ils servent aussi les besoins du registre lui-même, plus important peutêtre que la question du genre et du type de texte auquel nous avons affaire (conte ou science-fiction), et qui confine ici à l'absurde et au parodique.

\section{Mathématiques de l'absurde}

11 Si les personnages du feuilleton peuvent évoluer dans ce monde absurde, c'est parce qu'ils font eux-mêmes preuve d'une logique qui convient à leur environnement. C'est surtout vrai pour les Shadoks, jamais à court d'idées farfelues ${ }^{22}$, et dont les actions se résument à cette maxime: Ce n'est qu'en essayant continuellement qu'on finit par réussir. Ou en d'autres termes, plus ça rate, plus il y a de chance que ça marche. ${ }^{23} \mathrm{En}$ effet, les Shadoks sont des personnages très rationnels, menant toujours leurs raisonnements jusqu'au bout. Mais, soit que le postulat de départ soit faux, soit que le raisonnement lui-même se grippe, la conclusion est toujours absurde, à l'instar de celle 
tout juste citée. Absurde ne veut pas dire, ici, erroné. Une conclusion erronée, en effet, pourrait être possible. Une conclusion absurde, au contraire, défie entièrement les lois de la logique. Dans le premier cas, le téléspectateur pourrait être pris au piège; dans le second cas, au contraire, il sait pertinemment que la proposition n'a pas de sens. Si les théorèmes et maximes énoncés sont le plus souvent ponctués par la phrase "et réciproquement ", c'est bien parce que l'on peut les retourner sans craindre de les contredire.

Les maximes reviennent régulièrement dans le feuilleton, et servent à ponctuer ou à justifier les actions des personnages. Elles correspondent à deux traditions : la sentence traditionnelle d'une part, et le théorème mathématique de l'autre. Il s'agit donc d'un bon point de départ pour replacer le feuilleton dans une tradition de mouvements littéraires, artistiques et intellectuels centrée avant tout autour de la Pataphysique, et plus généralement des avant-gardes du XX siècle. Créée par Alfred Jarry à la fin du XIX ${ }^{\mathrm{e}}$ siècle, la 'Pataphysique est la science des solutions imaginaires. Parodiant les raisonnements scientifiques, elle met en avant «le principe de l'équivalence universelle et de la conversion des contraires ${ }^{24}$, un principe qui est à rapprocher du fameux «et réciproquement » cité plus haut. Son personnage le plus connu est Ubu-Roi, qui envoie à la trappe ses ennemis, tout comme le chef Shadok les envoie au «Goulp». La 'Pataphysique touche de nombreux artistes de la seconde moitié du $\mathrm{XX}^{\mathrm{e}}$ siècle qui intègrent son Collège: Raymond Queneau, Boris Vian, Ionesco, sont tous cités comme source d'inspiration par Jacques Rouxel. Ce dernier profite également du précédent créé par Jean-Christophe Averty, également membre du collège, et qui avait monté en 1965 une version d'Ubu Roi pour la télévision. Grâce à la 'Pataphysique, science, littérature et logique de l'absurde se trouvent liées. Ces prémices sont donc également présentes dans les courants surréalistes, chez les dadaïstes et les oulipiens, qui ont en commun le « refus de prendre les choses au sérieux ${ }^{25}$ et l'instauration d'un dialogue avec les sciences, et particulièrement les mathématiques. Les dadaïstes veulent éliminer toute logique formelle, particulièrement dans le langage. Ainsi, « le proverbe, le lieu commun, l'extrait typographique enclenchent une double combinatoire productrice de rêve et de réalité ${ }^{26}$. Tout comme pour les surréalistes, ce rapport à la langue enclenche un véritable travail sur la maxime. Plus tard, l'OuLiPo renoue à son tour avec le versant mathématique du langage en maniant des objets verbaux, sans sous-estimer, encore une fois, la place du rire. Au croisement entre l'OuLiPo et la 'Pataphysique, Raymond Queneau joue un rôle d'autant plus important que, en tant que mathématicien, il a également collaboré aux Éléments de mathématiques du collectif Bourbaki.

Or, Jacques Rouxel, lui-même mathématicien, se place autant dans la lignée des avantgardes que de Bourbaki, et il dit d'ailleurs s'être inspiré de la théorie des ensembles du groupe pour développer sa propre «théorie des ensembles passoiriques $»^{27}$. En d'autres termes, situé comme il l'était dans ce contexte scientifique et intellectuel, il ne pouvait qu'être sensible à l'absurde, digne hériter en quelque sorte de l'effervescence intellectuelle qui l'a précédé :

On appelle passoire tout instrument dans lequel on peut définir trois sousensembles : l'intérieur, l'extérieur, et les trous. L'intérieur est généralement placé au-dessus de l'extérieur, et se compose le plus souvent de nouilles et d'eau. Les trous ne sont pas importants. En effet, une expérience simple permet de se rendre compte que l'on ne change pas notablement les qualités de l'instrument en réduisant de moitié le nombre de trous, puis en réduisant cette moitié de moitié, etc, etc. Et à la 
limite jusqu'à ce qu'il n'y ait plus de trous du tout. D'où théorème, la notion de passoire est indépendante de la notion de trou, et réciproquement..$^{28}$

14 L'extrait ci-dessus est révélateur de la manière dont la science est traitée dans le feuilleton, agrémentée d'une logique de l'absurde qui fait feu de tout bois, se délectant du langage et s'appliquant aux éléments de la vie quotidienne (ici, les passoires, et plus loin les casseroles, et les autobus). Contrairement à la théorie initiale de Bourbaki, les sousensembles choisis dans l'épisode ne peuvent pas être décrits comme tels car ils n'ont pas d'existence propre hors de l'objet qu'ils forment. Plus exactement, ces sous-ensembles ainsi isolés et privés de raison d'être permettent de nier par l'absurde le postulat initial, soit l'idée qu'on ne change pas notablement les qualités de l'instrument en réduisant le nombre de trous. Dans le même temps, cette extrême formalisation du langage vient ici reprendre les reproches souvent faits à la théorie des ensembles, considérée comme inutilement verbeuse pour des applications simples. Ce genre de démonstration, non seulement par l'absurde, mais de l'absurde est ensuite repris dans le cours de géométrie, qui parodie les axiomes euclidiens, en partant de postulats tels que «le point est la plus courte distance possible entre deux lignes $»^{29}$. Les Shadoks sont en fait contemporains de la réforme dite des « maths modernes ", qui cherchait, en s'inspirant de Bourbaki, à réduire le poids des héritages culturels en formalisant au maximum l'enseignement secondaire. C'est donc là que Jacques Rouxel trouve également son inspiration pour sa propre "réforme des mathématiques »:

Le calcul a toujours donné beaucoup de fil à retordre aux Shadoks. En effet n'ayant que quatre cases il ne pouvait pas compter plus que quatre. Mais le professeur Shadoko avait réformé tout ça :

Quand il n'y a pas de Shadok, on dit GA, et on écrit ;

Quand il y a un Shadok de plus, on dit BU, et on écrit ;

Quand il y a encore un Shadok de plus, on dit ZO, et on écrit ;

Et quand il y en a encore un autre, on dit MEU, et on écrit.

Si je mets un Shadok en plus, évidemment, je n'ai plus assez de mots pour les compter... Alors c'est très simple : on les jette dans une poubelle, et je dis que j'ai BU poubelle. Et pour ne pas confondre avec le BU du début, je dis qu'il n'y a pas de Shadok à côté de la poubelle et j'écris BU GA. ${ }^{30}$ 
Figure 3. Le calcul en base 4, ou la mathématique des poubelles

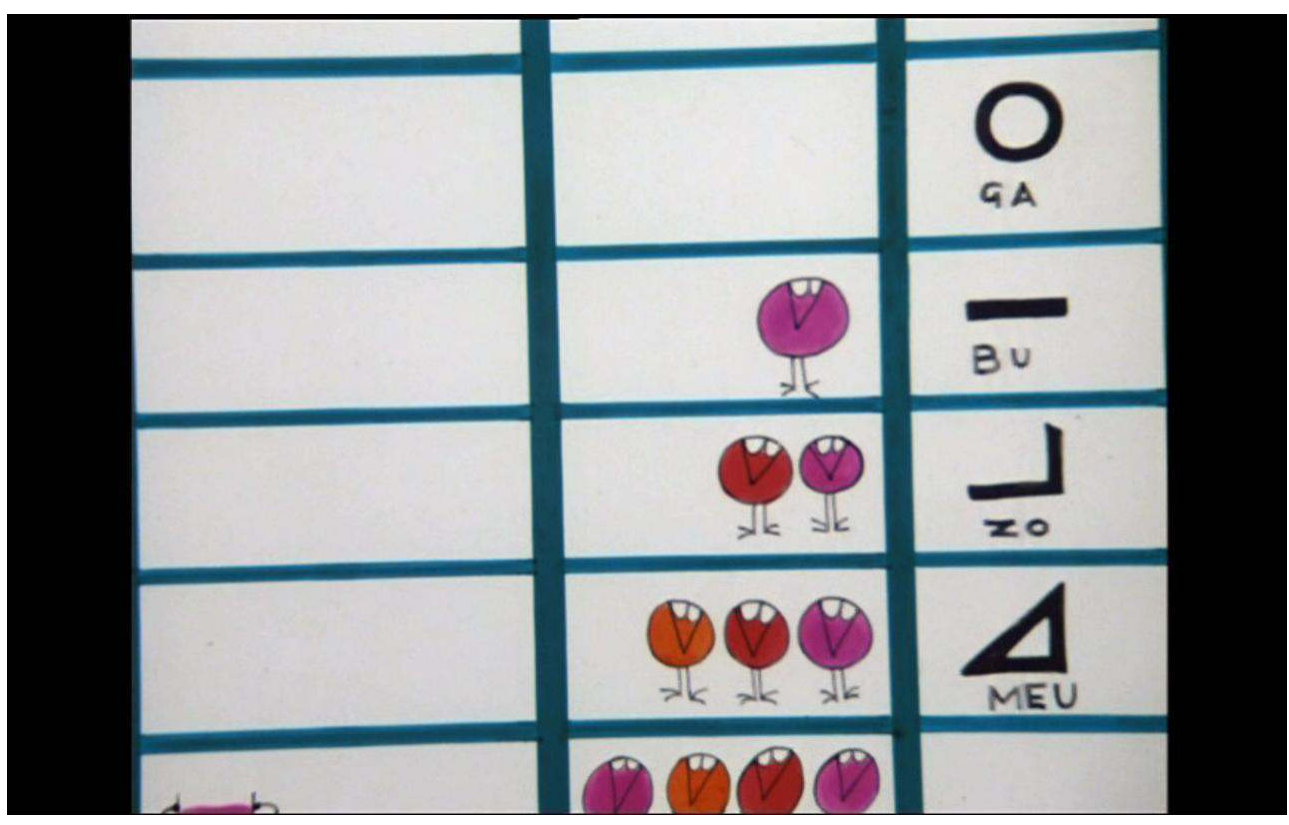

15 La logique de ce cours de calcul repose sur le système quaternaire, ou système de numération de base 4, qui utilise les chiffres $0,1,2$ et 3 pour représenter n'importe quel nombre réel. Mais au lieu d'utiliser des chiffres à proprement parler, les mathématiques Shadoks emploient des représentations d'eux-mêmes et des poubelles, autrement dit des objets issus de l'univers prosaïque du feuilleton. Qui plus est, il n'est pas innocent que le choix se porte sur une poubelle, qui est aussi un contenant dégradant, dans lequel on «jette » les Shadoks que l'on a fini de compter. Si une telle opération de calcul demande des connaissances scientifiques qui dépassent la culture scolaire classique, le rappel à la logique du récit - des Shadoks bêtes et méchants sans cesse malmenés par la narration raccroche ce nouveau type de savoir au monde de l'imaginaire. Le calcul en base 4, pour aussi scientifique qu'il soit, n'est en définitive qu'un nouveau moyen de se débarrasser des Shadoks.

\section{La science et ses gourous, source d'aliénation?}

16 Une telle insistance sur le caractère absurde de la science laisse toutefois matière à penser. Si les Shadoks sont éduqués, cette éducation fait fonctionner pleinement la logique de l'absurde, et n'est donc pas susceptible d'apporter savoir et progrès aux personnages. Pourtant, l'instruction est un motif important de la seconde série. L'idée naît en réponse aux plaintes des téléspectateurs, qui, confrontés au caractère absurde de la série, qualifiaient très souvent celle-ci, dans les courriers adressés à l'O.R.T.F., de profondément bête ${ }^{31}$. À une époque où la télévision, en tant que service public, se donne comme mission, outre d'informer et de divertir, d'instruire, c'est un reproche à prendre au sérieux, et en ce sens intégré dès le premier épisode de la nouvelle série par le narrateur:

C'est pourquoi, si nous avons cédé, malgré tout, devant l'insistance des Shadoks pour reparaître à partir d'aujourd'hui sur votre écran, c'est à la condition formelle et expresse qu'ils feraient un effort réel et sincère pour développer leur intelligence et relever le niveau culturel et éducatif de leur émission. Les Shadoks l'ont promis. ${ }^{32}$ 
Aussitôt, trois des Shadoks présents à l'écran prêtent serment en levant la patte. Mais le quatrième s'assomme avec son marteau : est-ce par bêtise, ou pour annoncer un futur "bourrage de crâne »? Toujours est-il que, comme nous l'avons dit, on retrouve à trois reprises les Shadoks sur les bancs de l'école, assistant à un cours magistral mené par le professeur Shadoko, le plus instruit des Shadoks, savant fou à l'origine de la plupart des inventions de la shadokologie (à commencer par la fusée qui leur permit de quitter leur planète). Systématiquement, la mise en scène de ses cours magistraux mêle bêtise des Shadoks et abrutissement dû à l'école. Ainsi leur inculque-t-on des "postulats bien gras » ${ }^{33}$, susceptibles, donc, de les étouffer, comme nous l'indique l'image présentant des bébés gavés au biberon et des adultes directement nourris de savoir à l'entonnoir.

Figure 4. Les Shadoks gavés au savoir

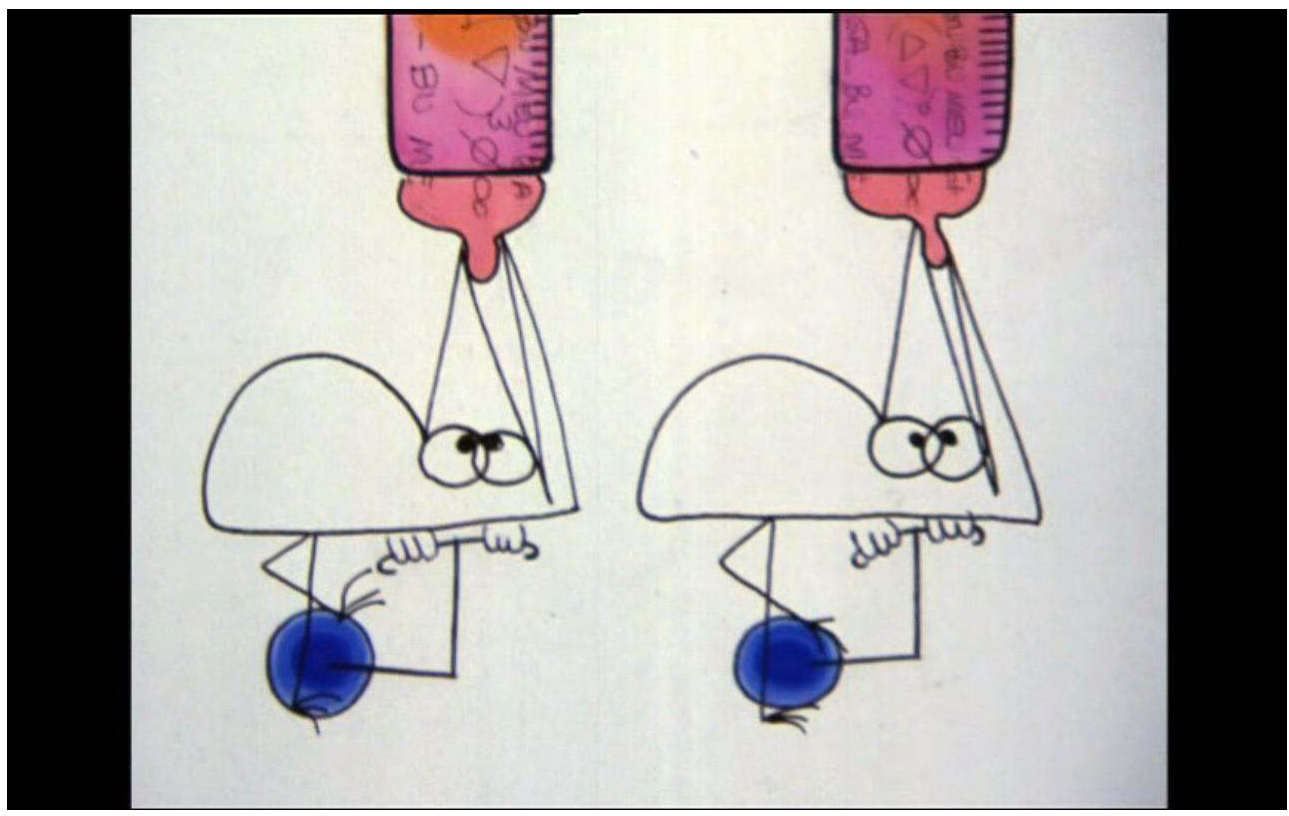

On allait la soumettre, la main-d'œuvre, à un régime spécial, sévère et accéléré, à base de calculs concentrés, de culture générale suractivée, de bons théorèmes fondamentaux, axiomes, postulats bien gras, et quantité de machins comme ça intellectuellement très nourrissants. ${ }^{34}$

L'éducation est aliénante parce qu'elle s'apparente plus à du gavage par la répétition incessante des mêmes mots qu'à un travail de la raison. De plus, elle est imposée par une volonté extérieure : celle des téléspectateurs, qui veulent des Shadoks intelligents, et, plus tard, celle des chefs, qui veulent pouvoir entreprendre des voyages cosmiques. Elle ne servira, en définitive qu'à " pomper intellectuellement ${ }^{35}$, c'est-à-dire à répéter indéfiniment la même action inutile qui occupe les Shadoks depuis le début du récit, action devenue désormais oxymorique, puisqu'elle est à la fois physique et intellectuelle.

La présence de chefs, dépositaires d'un certain savoir permet, en définitive, de définir ce rapport ambigu à la connaissance. Si le chef Shadok, qui, contrairement au Devin Plombier et au Professeur Shadoko, ne détient pas de savoir spécifique, les deux autres, en revanche, représentent chacun un aspect de la connaissance, l'une traditionnelle, l'autre moderne. 

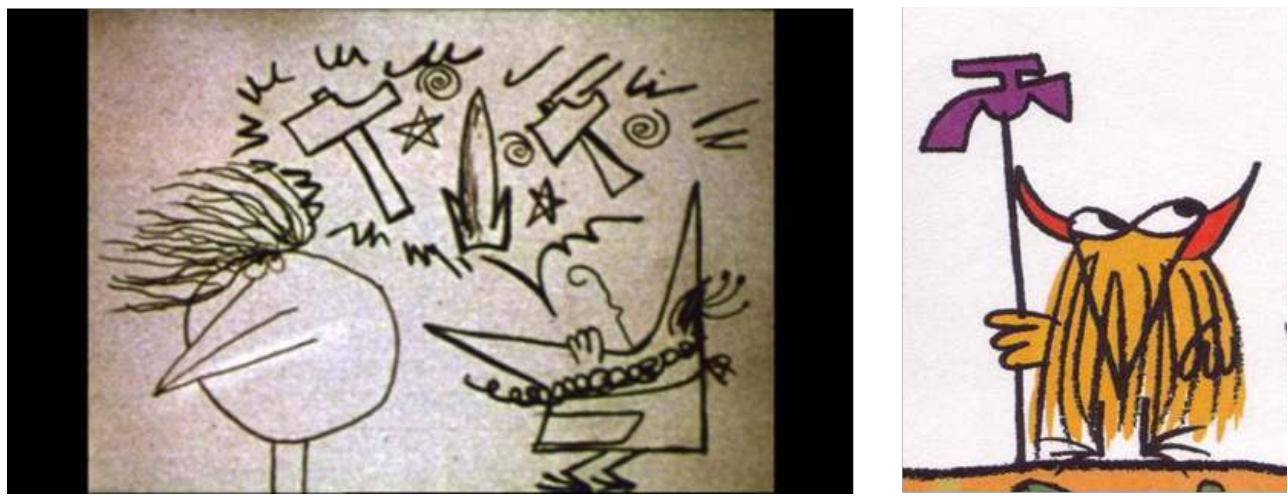

La représentation de ces chefs est symbolique, mais obéit à des stratégies de détournement qui mettent en valeur le caractère absurde de leur autorité. Le professeur Shadoko est doté d'une longue barbe qui, à elle seule, est supposée annoncer la sagesse du personnage. Mais celui-ci est pris, dès les premiers épisodes, en flagrant délit d'absence de maîtrise de lui-même, comme on peut le voir sur cette image issue de l'épisode 4 , où un simple Shadok est la cible placide de ses jurons. Le Devin Plombier, à la fois sorcier et chargé des problèmes de tuyauterie de la communauté Shadok, est quant à lui décrédibilisé par son nom même (qui fait de la sorcellerie un artisanat comme un autre), et par son insigne en forme de robinet, ne laissant en fin de compte à ses cornes rouges qu'un potentiel comique, et non plus diabolique. Ces figures de chef voient donc leur autorité minée par le récit et par le dessin, qui tous deux s'attachent à souligner leur potentiel comique.

Au-delà du caractère comique de leur représentation, le récit organise une confrontation conflictuelle entre ces deux chefs qui proposent des solutions différentes aux problèmes rencontrés par les Shadoks, qui cherchent à quitter la Lune et à revenir sur leur planète. Le Devin Plombier sort de son robinet la Vieille Légende, créature évidemment allégorique venue pour terroriser les Shadoks de manière irrationnelle en leur promettant la fin du monde s'ils ne pompent pas. Il fait donc appel à la superstition, pour revenir à une pratique traditionnelle, le pompage. Le professeur Shadoko, quant à lui, cherche au contraire à mettre en place les réformes de la science dont nous avons déjà parlées, pour pouvoir développer la technologie moderne, et améliorer l'astrolune, leur nouveau vaisseau. Leur conflit est mis en scène à la fin du dernier cours du Professeur Shadoko : «Tout le monde applaudissait très fort, sauf le Devin Plombier, qui disait qu'on n'avait pas idée d'inculquer à des enfants des bêtises pareilles, et que Shadoko il fallait le brûler. ${ }^{36}$ À travers ces deux personnages, la manie du passé comme celle du futur sont parodiées à part égales, l'une pour son conservatisme contre-productif et l'autre pour son obsession technologique. À ce titre, si le sorcier cherche à mettre le savant sur le bûcher, c'est aussi parce qu'il vient d'un autre temps où c'est le sort réservé aux hérétiques, ainsi qu'aux hommes de sciences qui dérangent l'ordre établi.

De fait, il semble qu'au-delà d'une simple comparaison, une hiérarchie s'opère entre ces savoirs, aussi imaginaires soient-ils. Dans la suite du récit, les Shadoks, éduqués pour améliorer l'astrolune, deviennent des «Shadoks à grosses têtes » : plus intelligents que leurs chefs, ils refusent de leur obéir, et, surtout, de pomper. Les chefs sont obligés de pomper à leur tour dans l'étage propulseur de l'astrolune, étage dont les Shadoks à grosses têtes se débarrassent une fois le décollage réussi ${ }^{37}$. Et le narrateur de conclure: 
«Depuis le temps qu'ils faisaient pomper les autres, après tout, ils n'ont que ce qu'ils méritent et notre histoire, de ce point de vue-là, n'est pas si immorale que ça. ${ }^{38}$ Cette conclusion confirme que le feuilleton a opéré, bien qu'en la basant sur l'humour, une dénonciation de l'aliénation dont sont coupables les figures d'autorité. C'est bien parce qu'il y a eu une dénonciation de celle-ci, que le récit a pu envisager d'en libérer ces personnages : le processus est performatif. Mais notons que c'est malgré tout grâce à leur éducation et au savoir que les Shadoks ont pu échapper à une situation aliénante, en prenant leur destin en main. Dans le conflit qui oppose le savoir traditionnel et le savoir moderne, c'est ce dernier qui l'emporte, car derrière la loufoquerie, il y a aussi une ode à la technologie et à la connaissance scientifique, tant pour son caractère moderne que pour son potentiel comique.

Sciences et savoirs imaginaires ont une place de premier choix dans Les Shadoks, un monde hyper-technologique inventé non pas tout à fait de toutes pièces, mais à partir des avancées scientifiques fascinantes des années soixante, en particulier en ce qui concerne l'espace. Si les objets de la science présentés sont parodiques, parfois trop prosaïques et toujours trop loufoques, il n'empêche qu'ils appartiennent à un ensemble de connaissances dont la transmission est possible. En ce sens, le savoir transmis au téléspectateur, pour aussi absurde qu'il soit, n'en reste pas moins primordial que la narration elle-même. Ceci explique que Jacques Rouxel ait privilégié autant d'épisodes "pause ", dont l'intégralité est consacrée à l'approfondissement de la connaissance du monde des Shadoks. Paradoxalement, ce sont aussi les épisodes les moins appréciés par les téléspectateurs, peut-être parce qu'ils demandent un trop grand effort de crédulité, sans proposer de récit comme support aux mécanismes de l'imaginaire. De fait, la transmission du savoir shadokologique est impossible si le téléspectateur n'accepte pas d'adhérer entièrement à une logique qui n'existe que dans le feuilleton, mais ce faisant, il accède en réalité à un faisceau de savoirs qui dépasse largement le simple univers de la série, puisque celle-ci s'appuie sur des paradigmes existants et développés en dehors d'elle, logique des avant-gardes et de la philosophie mathématique. Par conséquent, bien que soit souligné le danger de l'aliénation complète du savoir, qui est toujours potentiellement un savoir imaginaire au service d'une autorité malveillante, Les Shadoks demeurent, en fin de compte, une ode à la connaissance, si celle-ci reste au service de l'imaginaire.

\section{NOTES}

1. «On ne peut pas faire d'animation en 1960 sans avoir englouti, assimilé toutes les fluctuations de l'art moderne, l'art abstrait, le tachisme, le futurisme et le surréalisme », Robert Benayoun, Le dessin animé après Walt Disney, Paris, Jean-Jacques Pauvert, 1961, p. 7.

2. On appelle communément le style de Walt Disney le « O-Style », et celui de l'école de Bosustow, inspiré du trait de Saul Steinberg, le «I-Style ».

3. Nous reprenons ici les termes de P. Vallotton, qui explique que la notion de «culture médiatique " est venue remplacer et désidéologiser celle de "culture de masse " chez les historiens actuels : "Cette expression met l'accent sur les nouveaux modes de circulation des 
discours et des productions culturelles liés à la démultiplication des supports. », Philipe Valotton, «Culture médiatique», in Dictionnaire d'histoire culturelle de la France contemporaine, Paris, PUF, 2010, p. 219.

4. «Futurisme », Trésor de la langue française informatisé, http://atilf.atilf.fr/tlf.htm (consulté le $1^{\mathrm{er}}$ octobre2014).

5. «Science-fiction", Trésor de la langue française informatisé, http://atilf.atilf.fr/tlf.htm (consulté le $1^{\text {er }}$ octobre 2014).

6. «La science-fiction n'est pas une chose unique. C'est plutôt toute une somme de choses - une installation dans le futur, une machine merveilleuse, une société idéale, une créature extraterrestre, un voyage dans le temps, un voyage interstellaire, une perspective satirique, une approche particulière du contenu narratif, et tout ce que nous recherchons quand nous recherchons de la science-fiction, ici avec évidence, ailleurs plus subtilement - choses qui sont tressées ensemble en une variété infinie de combinaisons. " Kincaid Paul, «On the Origins of Genre », Extrapolation, n 44, hiver 2003, p. 409-419, cité dans John Rieder, "On Defining SF, or Not: Genre Theory, SF, and History ", in Science Fiction Studies, vol. 37, $\mathrm{n}^{\circ} 116$, Novembre 2010, p. 191-209, traduit par Irène Langlet http://resf.revues.org/489?lang=en (consulté le 30 janvier 2015).

7. John Rieder, « On Defining SF, or Not : Genre Theory, SF, and History », in Science Fiction Studies, vol. 37, n 116, novembre 2010, p. 191-209

8. Série 1, épisode 1 . Nous soulignons.

9. Série 1 , épisode 44.

10. Série 2, épisode 4.

11. Série 2, épisode 2.

12. Série 2, épisode 39.

13. Série 2, épisode 15.

14. INA, fonds Jacques Durand, versement 2006, fonds O.R.T.F. opinions 1968-1971, service des études des marchés sur Les Shadoks.

15. Saison 2, épisode 26.

16. Respectivement série 2, épisodes 13-16 et série 1 , épisode 7.

17. Série 1 , épisode 3.

18. Respectivement série 1, épisode 17 et série 2, épisode 15.

19. Série 1, épisode 1. Les Shadoks du bas ont les pieds tournés vers le ciel afin de soutenir leur planète par en bas pour éviter qu'elle ne tombe.

20. Série 1, épisode 1.

21. Série 2, épisode 50.

22. Pour faire avancer la Shadokaravelle quand il n'y a plus d'eau, il suffit, par exemple, de prendre de l'eau à l'arrière du bateau et de la rejeter à l'avant.

23. Série 1, épisode 18.

24. Roger Shattuck, Au seuil de la 'Pataphysique, Collège de 'Pataphysique, texte doctrinal présenté en 9 langues, 1962, p. 19.

25. Henri Behar et Michel Carassou, Dada, histoire d'une subversion, Paris, Fayard, 1990, p. 137.

26. Ibid, p. 72.

27. Jacques Rouxel, INA, Tous les Mickeys du monde, France Inter, 18 août 2007.

28. Série 2, épisode 6.

29. Série 2, épisode 44 .

30. Série 2, épisode 44.

31. "Une émission loufoque, enfantée par des corniauds, ajoutons même des débiles mentaux ", assène un homme de Saint-Brieuc qui écrit le 28/03/1969 [A.A.A Productions, Courrier des téléspectateurs, 1968-1972].

32. Série 2, épisode 1. 
33. Série 2, épisode 40.

34. Série 2, épisode 40.

35. Série 2, épisode 40.

36. Série 2, épisode 44.

37. Série 2 , épisodes 44 à 47.

38. Série 2, épisode 47.

\section{RÉSUMÉS}

Créé par Jacques Rouxel, le dessin-animé des Shadoks inscrit l'imaginaire français de la fin des années 1960 dans un contexte technologique et artistique résolument moderne. Sciences et savoirs imaginaires y ont une place de premier choix, dans une sorte de genèse «futuriste » qui s'inspire des avancées techniques des années 1960 et parodie de manière farfelue les savoirs scientifiques, théoriques et mathématiques, en s'inspirant par exemple de la 'Pataphysique. Toutefois, le feuilleton traite avant tout de l'absurdité possible de la science, jusqu'à poser la question du caractère aliénant de celle-ci, notamment lorsqu'elle est au service de l'autorité. En définitive, les Shadoks ne célèbrent la connaissance que si celle-ci reste au service de l'imaginaire.

\section{INDEX}

Mots-clés : cinéma, série, Shadoks (Les), science

\section{AUTEUR}

JESSICA KOHN 